\title{
Plaque Regression Determined by Intravascular Ultrasound Predicts Long-Term Outcomes of Patients with Acute Coronary Syndrome
}

\author{
Tomotaka Dohi, Katsumi Miyauchi, Shinya Okazaki, Takayuki Yokoyama, Naotake Yanagisawa, \\ Hiroshi Tamura, Takahiko Kojima, Ken Yokoyama, Takeshi Kurata, and Hiroyuki Daida
}

Department of Cardiovascular Medicine, Juntendo University School of Medicine, Tokyo, Japan

\begin{abstract}
Aim: The usefulness of drugs to treat plaque regression is assessed by intravascular ultrasound (IVUS); however, the impact of plaque regression on clinical outcomes in patients with acute coronary syndrome (ACS) has not been established; therefore, we investigated the relationship between coronary plaque regression and long-term clinical outcomes.

Methods: We analyzed data from 86 patients who underwent percutaneous coronary intervention (PCI) and who were assessed in detail at baseline and at 6 months of follow-up by measuring proximal non-culprit sites of PCI lesions using volumetric IVUS. Patients were divided according to changes in plaque volume over 6 months into one group with plaque regression $(n=55 ; 64.0 \%)$ and another with progression $(n=31 ; 36.0 \%)$. They were followed up observationally for a mean of 1,736 days.

Results: Baseline characteristics at the time of ACS were similar between the groups. The probability of event-free survival was significantly higher in the regression group than in the progression group as estimated by the Kaplan-Meier method (Log-rank test, $p=0.032$ ). Furthermore, the Cox hazards model revealed the relative contribution of plaque regression as a predictor of cardiovascular events (hazard ratio: $0.26 ; 95 \% \mathrm{CI}, 0.07$ to $0.83 ; p=0.023$ ).

Conclusions: Plaque regression determined by volumetric IVUS over a period of 6 months was associated with a lower rate of cardiovascular events among patients with ACS. This study also demonstrated that plaque regression could be a surrogate marker of future cardiovascular events.
\end{abstract}

J Atheroscler Thromb, 2011; 18:231-239.

Key words; Plaque regression, Prognosis, Acute coronary syndrome, Atherosclerosis

\section{Introduction}

Data derived from intravascular ultrasound (IVUS) have provided important insights into the progression of atherosclerosis and remodeling in the arterial wall ${ }^{1,2)}$. In addition, volumetric findings of plaque change using IVUS have served as a clinical endpoint of pharmacological interventions in clinical trials ${ }^{3-8}$. Furthermore, IVUS meta-analysis has demonstrated that statins promote significant coronary plaque regression as assessed by IVUS ${ }^{9}$. The validity

Address for correspondence: Katsumi Miyauchi, Department of Cardiovascular Medicine, Juntendo University School of Medicine, 2-1-1 Hongo, Bunkyo-ku, Tokyo 113-0033, Japan

E-mail: ktmmy@med.juntendo.ac.jp

Received: July 25, 2010

Accepted for publication: October 8, 2010 of plaque regression as an endpoint in clinical trials would be enhanced if a close association between regression and future clinical events could be illustrated; however, the relationship between plaque burden determined by IVUS and future cardiovascular events has not been defined.

The ESTABLISH trial demonstrated a beneficial effect of atorvastatin on plaque regression over a period of 6 months in patients with acute coronary syndrome $(\mathrm{ACS})^{10)}$. We extended the study of the recruited patients and applied the same protocol as the ESTABLISH trial to verify the effect of atorvastatin or changes in coronary plaque on long-term clinical outcomes of patients with ACS. That study was referred to as the Extended-ESTABLISH trial ${ }^{11)}$. Overall, little evidence has indicated how changes in plaque volume, such as progression or regression, influence clinical 
outcomes after ACS. Thus, the present study investigated whether short-term plaque regression can become a surrogate endpoint and long-term predictor of clinical outcomes in patients with ACS.

\section{Methods}

\section{Study Protocol}

This observational cohort study included patients with ACS who were treated with PCI and underwent a complete sequential IVUS evaluation over a period of 6 months (the Extended-ESTABLISH: IVUS study). The objective was to assess the long-term clinical impact of changes in coronary plaque. The Extended-ESTABLISH trial, which was an extended version of the ESTABLISH trial, has been described in detail ${ }^{10-11)}$. The Extended-ESTABLISH follow-up $(n=180)$ demonstrated that starting atorvastatin therapy within $48 \mathrm{~h}$ of ACS onset followed by intensive lipid-lowering therapy for 6 months is associated with a reduced incidence of long-term cardiovascular events in patients with ACS after PCI.

The inclusion criteria for the present study was a change in the proximal site from an ACS culprit lesion that could be precisely measured by IVUS. We considered that plaque on the proximal side of a coronary artery leads to more adverse cardiac events than that on the distal side in clinical practice ${ }^{12}$. We also considered that plaque evaluation on the proximal side is more useful than that on the distal side for PCI under IVUS guidance, because taking the IVUS catheter to the distal side is more complicated and invasive during acute-phase treatment. Thus, this study estimated the impact of plaque volume, particularly in the proximal coronary artery segment, on long-term clinical outcomes.

We defined regression as a decrease or no change in plaque volume from baseline, and progression as an increase in the amount of plaque. Patients were assigned to either a regression or progression group and their long-term clinical outcomes from the viewpoint of follow-up IVUS were compared. Structured lipid management of all patients was discontinued after an IVUS examination at 6 months; thereafter, the attending physicians administered all patients with statins to achieve and maintain LDL-C values as close to $<100 \mathrm{mg} / \mathrm{dL}$ as possible ${ }^{13)}$. All baseline data were used at the time of ACS onset in each patient. Our institutional review board approved this study and each patient provided written informed consent to participate in the follow-up.

\section{End Points}

The primary endpoint was the first occurrence of major adverse cardiac and cerebrovascular events (MACCE), namely, all-cause death, recurrent ACS and stroke. We defined ACS as high-risk unstable angina, non-ST-elevated myocardial infarction (MI) or ST-elevated MI. An increase ( $\geq 2$-fold) in serum creatine phosphokinase and troponin $\mathrm{T}$ positivity indicated a diagnosis of MI. Moreover, recurrent ACS was defined as AMI and unstable angina requiring emergency hospitalization for either PCI or coronary artery bypass grafting. Stroke was diagnosed based on the presence of a neurologic deficit that was confirmed by computed tomography or magnetic resonance imaging.

Outcome data were collected by serial contact with the patients or their families until March 2008. The medical records of patients who died or who were treated at our hospital were analyzed. Other institutions that admitted patients provided details and causes of MACCE.

\section{IVUS Examination and Analysis}

All IVUS images were acquired as described using a $40-\mathrm{MHz}, 2.9 \mathrm{~F}$ system (Boston Scientific) at baseline and follow-up ${ }^{10)}$. After the intracoronary administration of nitroglycerin $(0.2 \mathrm{mg})$, an ultrasound catheter was positioned $\geq 10 \mathrm{~mm}$ distal to the PCI site. The catheter was automatically retracted at $0.5 \mathrm{~mm} / \mathrm{s}$ and IVUS measurements were recorded on super VHS videotape and quantified offline. Plaque volume was assessed by volumetric analysis using a Netra 3D IVUS system (ScImage, CA, USA). Baseline and follow-up IVUS images were reviewed side-byside on a display and target segments were selected. One target segment was determined at a non-PCI site that was $>5 \mathrm{~mm}$ proximal to the PCI site with a reproducible index side branch. Plaque was measured to determine those that were as close to $10 \mathrm{~mm}$ in length as possible. Segments with obvious calcification or tortuosity were avoided. An independent experienced IVUS investigator who was blinded to the patient groups and angiographic results quantified data that included vessel, lumen and plaque volumes. Standard measurements included lesion length, vessel and lumen volumes. Plaque volume was calculated as vessel volume minus lumen volume. The \%change in plaque volume was defined as a change in plaque volume (follow-up minus baseline plaque volume) divided by baseline plaque volume.

\section{Quantitative Coronary Analysis}

All coronary angiographic images were analyzed 
Table 1. Baseline characteristics of patients and additional therapy during follow-up

\begin{tabular}{|c|c|c|c|c|}
\hline & All patients $(n=86)$ & Regression group $(n=55)$ & Progression group $(n=31)$ & $p$ value \\
\hline Age (y) & $62.9 \pm 9.0$ & $63.0 \pm 10.0$ & $62.8 \pm 8.6$ & 0.907 \\
\hline Men, n (\%) & $71(82.5)$ & $45(81.8)$ & $26(83.8)$ & 0.801 \\
\hline Body mass index, $\mathrm{kg} / \mathrm{m}^{2}$ & $24.2 \pm 3.2$ & $24.5 \pm 2.7$ & $24.1 \pm 3.5$ & 0.808 \\
\hline Hypertension, n (\%) & $50(58.1)$ & $35(63.6)$ & $15(48.4)$ & 0.169 \\
\hline Systolic blood pressure (mmHg) & $139.1 \pm 23.7$ & $139.2 \pm 24.8$ & $139.0 \pm 22.3$ & 0.996 \\
\hline Diastolic blood pressure $(\mathrm{mmHg})$ & $77.2 \pm 15.0$ & $78.3 \pm 15.6$ & $74.9 \pm 13.8$ & 0.315 \\
\hline Diabetes, n (\%) & $34(39.5)$ & $20(36.4)$ & $14(45.1)$ & 0.424 \\
\hline Smoker, n (\%) & $54(62.8)$ & $35(63.6)$ & $19(61.3)$ & 0.829 \\
\hline Prior CAD, n (\%) & $15(17.4)$ & $6(10.9)$ & $9(29.0)$ & 0.037 \\
\hline Family history of CAD, n (\%) & $27(31.4)$ & $15(27.3)$ & $12(38.7)$ & 0.276 \\
\hline Classification of ACS, n (\%) & & & & 0.658 \\
\hline AMI, n (\%) & $56(65.1)$ & $34(61.8)$ & $22(70.9)$ & \\
\hline Unstable angina, n (\%) & $35(34.9)$ & $21(38.2)$ & $9(29.0)$ & \\
\hline Culprit lesion of ACS, n (\%) & & & & 0.577 \\
\hline Left anterior descending artery & $42(48.9)$ & $26(47.3)$ & $16(51.6)$ & \\
\hline Left circumflex artery & $16(18.6)$ & $12(21.8)$ & $4(12.9)$ & \\
\hline Right coronary artery & $28(32.6)$ & $17(30.9)$ & $11(35.5)$ & \\
\hline Maximum CPK, IU/L & $1638.7 \pm 1908.5$ & $1692.2 \pm 1988.6$ & $1551.5 \pm 1795.4$ & 0.635 \\
\hline Atorvastatin therapy, n (\%) & $40(46.5)$ & $34(61.8)$ & $6(19.4)$ & $<0.0001$ \\
\hline ACE inhibitor, n (\%) & $41(48.8)$ & $26(49.1)$ & $15(48.4)$ & 0.952 \\
\hline AT1 antagonist, n (\%) & $31(36.9)$ & $18(34.0)$ & $13(41.9)$ & 0.483 \\
\hline$\beta$-Blockers, $\mathrm{n}(\%)$ & $45(53.6)$ & $29(54.7)$ & $16(51.6)$ & 0.324 \\
\hline
\end{tabular}

$\mathrm{CAD}$, coronary artery disease; ACS, acute coronary syndrome; AMI, acute myocardial infarction; CPK, creatine phosphokinase; AT1, angiotensin receptor type 1 . Values are the means \pm SD where appropriate. Categorical data analyzed by $\chi^{2}$ test; continuous data analyzed by unpaired Student's $t$ test or Mann-Whitney rank-sum test. $p<0.05$ was considered significant.

at our institution using a Cardiovascular Angiography Analysis System II (CAAS II; Pie Medical Imaging, Maastricht, The Netherlands). Experienced technicians supervised by an expert physician performed quantitative coronary analysis (QCA) of matched projections from baseline and follow-up coronary angiographic images. Reference sites were measured in every patient.

\section{Statistical Analysis}

Continuous variables are expressed as the means \pm SD. Data from two independent groups were compared using a $t$-test or the Wilcoxon rank-sum test and intra-group data were analyzed using a paired $t$-test or the Wilcoxon signed-rank test. Categorical data were tabulated as frequencies and percentages and compared using the $\chi^{2}$ test or Fisher's exact test. Event-free survival probabilities for MACCE were estimated using the Kaplan-Meier method and group differences were assessed using a log-rank test. Hazard ratios for each variable were calculated using a Cox proportional hazards model that included plaque regression, age, gender, BMI, hypertension, diabetes and type of ACS as variables. A two-sided $p$-value of $<0.05$ was considered significant. All data were analyzed using JMP version 7.0 for Windows (SAS Institute, Cary, NC).

\section{Results}

\section{Characteristics of Study Subjects}

Of the 114 patients who completed IVUS twice during the Extended-ESTABLISH trial, 86 (75.4\%) were included in the present study. We excluded 28 patients $(24.6 \%)$ if they had no adequate plaque lesions to evaluate (insufficient plaque, too short at the proximal site or poor IVUS imaging quality). Accordingly, we assessed changes in plaques using IVUS in 86 patients (mean age, 62.9 \pm 9.0 years; male, $n=71 ; 82.5 \%$ ) with ACS who underwent percutaneous coronary intervention. Plaques regressed and progressed in $55(64.0 \%)$ and $31(36.0 \%)$ of these patients, respectively. Baseline features at the time of ACS occurrence were similar between the two groups. Factors related to ACS, such as classification and culprit vessels, did not significantly differ between the groups. 
Table 2. Baseline and follow-up intravascular ultrasound and angiographic findings

\begin{tabular}{|c|c|c|c|c|c|c|}
\hline & \multicolumn{3}{|c|}{ Regression group $(n=55)$} & \multicolumn{3}{|c|}{ Progression group $(n=31)$} \\
\hline \multicolumn{7}{|l|}{ IVUS measurement } \\
\hline Total length, mm & $89.2 \pm 24.2$ & $89.0 \pm 22.6$ & 0.322 & $84.5 \pm 24.2$ & $85.2 \pm 22.9$ & 0.324 \\
\hline Vessel volume, $\mathrm{mm}^{3}$ & $180.4 \pm 64.7$ & $170.5 \pm 64.2$ & $<0.001$ & $156.6 \pm 51.2$ & $160.0 \pm 47.4$ & 0.416 \\
\hline Change in vessel volume, $\mathrm{mm}^{3}$ & & $-9.9 \pm 15.8^{*}$ & & & $3.3 \pm 17.7$ & \\
\hline Change in lumen volume, $\mathrm{mm}^{3}$ & & $0.4 \pm 14.9$ & & & $-4.1 \pm 16.2$ & \\
\hline Change in lumen volume (\%) & & $-4.1 \pm 15.9$ & & & $-7.6 \pm 21.1$ & \\
\hline Plaque volume, $\mathrm{mm}^{3}$ & $85.1 \pm 38.0$ & $75.5 \pm 35.9$ & $<0.001$ & $76.5 \pm 36.9$ & $83.8 \pm 37.7$ & $<0.001$ \\
\hline Percent plaque volume, $\%$ & $46.9 \pm 11.8$ & $44.6 \pm 13.2$ & $<0.001$ & $47.2 \pm 13.4$ & $51.0 \pm 14.1$ & $<0.001$ \\
\hline Change in plaque volume, $\mathrm{mm}^{3}$ & & $-9.6 \pm 7.2^{*}$ & & & $7.4 \pm 6.5$ & \\
\hline \multicolumn{7}{|l|}{ Quantitative Coronary Angiography } \\
\hline Percent change QCA, \% & & $-7.8 \pm 9.4$ & & & $-9.0 \pm 10.7$ & \\
\hline
\end{tabular}

IVUS, intravascular ultrasound; QCA, quantitative coronary angiography. Values are the means \pm SD where appropriate. Unpaired Student's $t$ test or Mann-Whitney rank-sum test was used to analyze continuous data. $p<0.05$ was considered significant. Regression vs. progression group: ${ }^{*} p<0.01$.

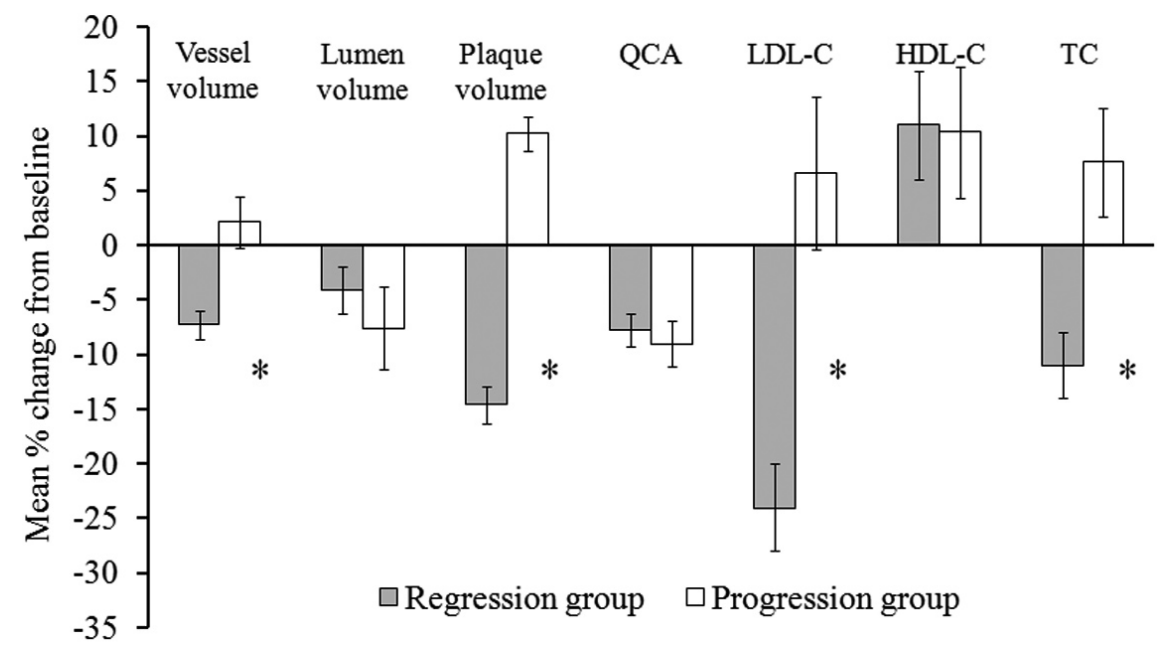

Fig. 1. Mean \% change in IVUS results and lipid profile.

Plaque and vessel volumes significantly decreased in regression group, whereas the number of vessels increased with increasing plaque volume in progression group. Percent changes in LDL-C from baseline in regression and progression groups were $-24.0 \%$ and $6.6 \%$, respectively. However, \% change in HDL-C did not significantly differ between groups. Regression vs. progression, ${ }^{*} p<0.05$.

More patients were assigned to receive atorvastatin (20 $\mathrm{mg} /$ day) during the first 6 months in the ExtendedESTABLISH trial in the regression group than in the progression group $(n=34,61.8 \%$ vs. $n=6,19.4 \%$, $p<0.001$ ) (Table 1). All patients in both groups were treated after 6 months with statins, and the dose and type (including atorvastatin, pitavastatin, pravastatin, simvastatin and fluvastatin) were appropriately changed for optimal lipid management. At 6 months after the IVUS study (that is, 1 year after ACS onset), statins were administered to both groups at the same frequency $(86.7 \%$ vs. $84.3 \%, p=0.762)$. At that time, the mean LDL-C values were $87.8 \pm 23.4$ and $97.4 \pm$ $20.4 \mathrm{mg} / \mathrm{dL}$, respectively $(p=0.031)$. 
Table 3. Blood parameters of patients at baseline and follow-up

\begin{tabular}{|c|c|c|c|c|c|c|}
\hline Laboratory parameters & \multicolumn{3}{|c|}{ Baseline } & \multicolumn{3}{|c|}{ Follow-up } \\
\hline Change in TC, \% & & & & $-11.0 \pm 24.4$ & $7.6 \pm 27.7$ & 0.002 \\
\hline HDL-C, mg/dL & $45.8 \pm 12.2$ & $43.1 \pm 15.9$ & 0.112 & $49.3 \pm 12.5^{*}$ & $49.8 \pm 23.2^{*}$ & 0.283 \\
\hline Change in LDL-C, \% & & & & $-24.0 \pm 31.2$ & $6.6 \pm 42.9$ & 0.001 \\
\hline Change in HDL-C, \% & & & & $11.0 \pm 19.8$ & $10.3 \pm 18.3$ & 0.371 \\
\hline LDL-C/HDL-C ratio & $2.87 \pm 1.01$ & $2.88 \pm 1.05$ & 0.804 & $1.96 \pm 0.97^{\dagger}$ & $2.63 \pm 1.11$ & 0.009 \\
\hline Apolipoprotein A1, mg/dL & $113.7 \pm 16.9$ & $110.2 \pm 28.4$ & 0.096 & $129.5 \pm 20.5^{\dagger}$ & $125.2 \pm 25.1^{\dagger}$ & 0.158 \\
\hline Apolipoprotein B, mg/dL & $88.3 \pm 20.0$ & $89.6 \pm 18.2$ & 0.784 & $77.4 \pm 25.3^{\dagger}$ & $95.0 \pm 21.2$ & 0.003 \\
\hline hsCRP, mg/dL & $0.96 \pm 1.72$ & $1.05 \pm 2.58$ & 0.111 & $0.12 \pm 0.17^{*}$ & $0.18 \pm 0.31^{*}$ & 0.373 \\
\hline
\end{tabular}

TC, total cholesterol; HDL-C, high-density lipoprotein; LDL-C, low-density lipoprotein; Apo B, apolipoprotein B; Apo A1, apoprotein A1; and hsCRP, high-sensitivity C-reactive protein. Values are the means \pm SD when appropriate. The $\chi^{2}$ test was used for categorical data and unpaired Student's $t$ test or Mann-Whitney rank-sum test for continuous data. $p<0.05$ was considered significant. Baseline vs. follow-up: ${ }^{*} p<0.05$; $p<0.01$.

\section{Changes in IVUS Findings, QCA and Lipid Profiles} Plaque and vessel volumes significantly decreased from $85.1 \pm 38.0$ to $75.5 \pm 35.9 \mathrm{~mm}^{3}$ ( $p<0.001$ vs. baseline) and from $180.4 \pm 64.7$ to $170.5 \pm 64.2 \mathrm{~mm}^{3}$ $(p<0.001)$ respectively, in the regression group. In contrast, total vessel volume within the external elastic membrane increased from $156.6 \pm 51.2$ to $160.0 \pm$ $47.4 \mathrm{~mm}^{3}(p=0.416)$ with increasing plaque volume from $76.5 \pm 36.9$ to $83.8 \pm 37.7 \mathrm{~mm}^{3}(p<0.001)$ in the progression group. Lumen volume tended to decrease in both groups, but the difference did not reach significance (regression, from $95.4 \pm 41.2$ to $95.0 \pm 43.8$ $\mathrm{mm}^{3}, p=0.923$; progression, from $80.2 \pm 27.7$ to 76.1 $\left.\pm 26.8 \mathrm{~mm}^{3}, p=0.103\right)$. However, the reference diameter was significantly decreased in the QCA analysis of both groups (regression, from $3.14 \pm 0.29$ to $2.90 \pm$ $0.44 \mathrm{~mm}, p<0.001$; progression, from $3.12 \pm 0.50$ to $2.88 \pm 0.53 \mathrm{~mm}, p<0.001$ ) (Table 2 and Fig. 1).

The level of LDL-C fell to a mean of $91.4 \pm 37.9$ $\mathrm{mg} / \mathrm{dL}$ (median, $85.4 \mathrm{mg} / \mathrm{dL}$ ) in the regression group at follow-up IVUS $(p<0.001)$, but reached a mean of $113.6 \pm 31.4 \mathrm{mg} / \mathrm{dL}$ (median, $119.6 \mathrm{mg} / \mathrm{dL}$ ) in the progression group $(p=0.930)$. Furthermore, the \%changes in LDL-C from baseline in the regression and progression groups were $-24.0 \pm 31.2 \%$ and 6.6 $\pm 42.9 \%$, respectively $(p=0.001)$. The ratios of LDL$\mathrm{C} / \mathrm{HDL}-\mathrm{C}$ and of ApoB/ApoA1 were significantly lower in the regression group than in the progression group $(1.96 \pm 0.97$ vs. $2.63 \pm 1.11, p=0.009$ and 0.61 \pm 0.23 vs. $0.78 \pm 0.24, p=0.002$, respectively; Table 3).

\section{Clinical Events and Multivariate Analysis}

Prognostic data were fully documented during the entire follow-up period (mean duration, 1736 589 days), during which MACCE developed in five (death, $n=2$; ACS, $n=3$ ) patients in the regression group and in eight (death, $n=4$; ACS, $n=3$; stroke, $n=1$ ) in the progression group (Fig. 2). Six patients developed ACS during the follow-up period, although it was not associated with the position of the measured plaque. None of the six patients with recurrent ACS had obvious stent thrombosis and three developed an obvious clinical presentation and events associated with the index stent procedure (2 of 3 in the regression group and 1 of 3 in the progression group). The remaining three patients developed new-onset ACS in a different vessel from that in which plaque was measured. The plaque volume changed significantly less in patients who developed MACCE than in those who did not $\left(94.8 \pm 31.9\right.$ to $95.7 \pm 29.2 \mathrm{~mm}^{3}, p=0.763$; $79.7 \pm 38.3$ to $75.4 \pm 37.0 \mathrm{~mm}^{3}, p=0.001$ ). Cumulative event-free survival was significantly higher in the regression group than in the progression group $(p=0.032$; log-rank test; Fig. 3).

The results of analysis using the unadjusted Cox proportional hazards model showed that plaque 


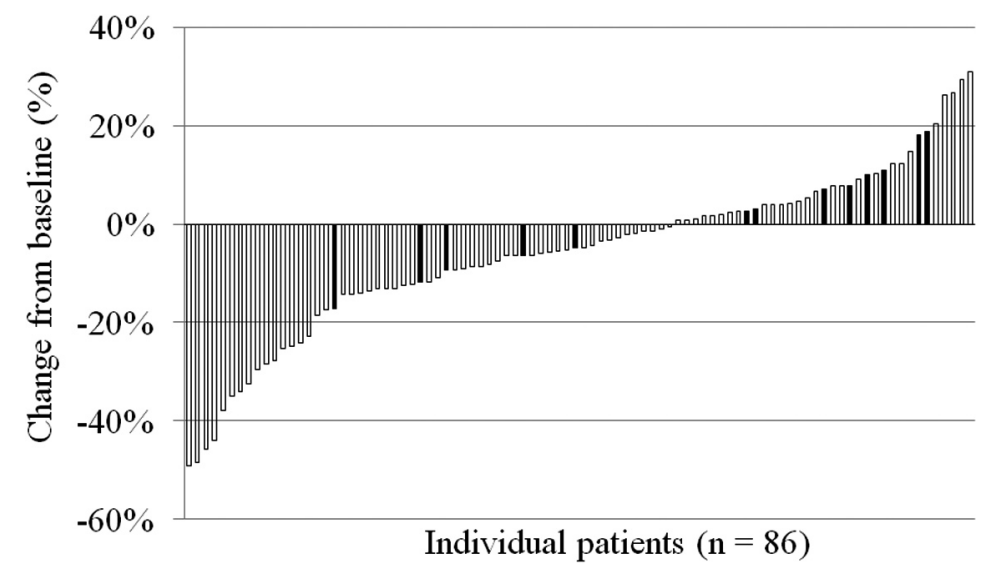

Fig. 2. Change in plaque volume from baseline using IVUS and incidence of MACCE.

Plaques regressed in $64 \%$ of patients after ACS. More patients died or developed cardiovascular events (Black bars) in progression group $(25.8 \%)$ than in regression group $(9.1 \%)$ during follow up.

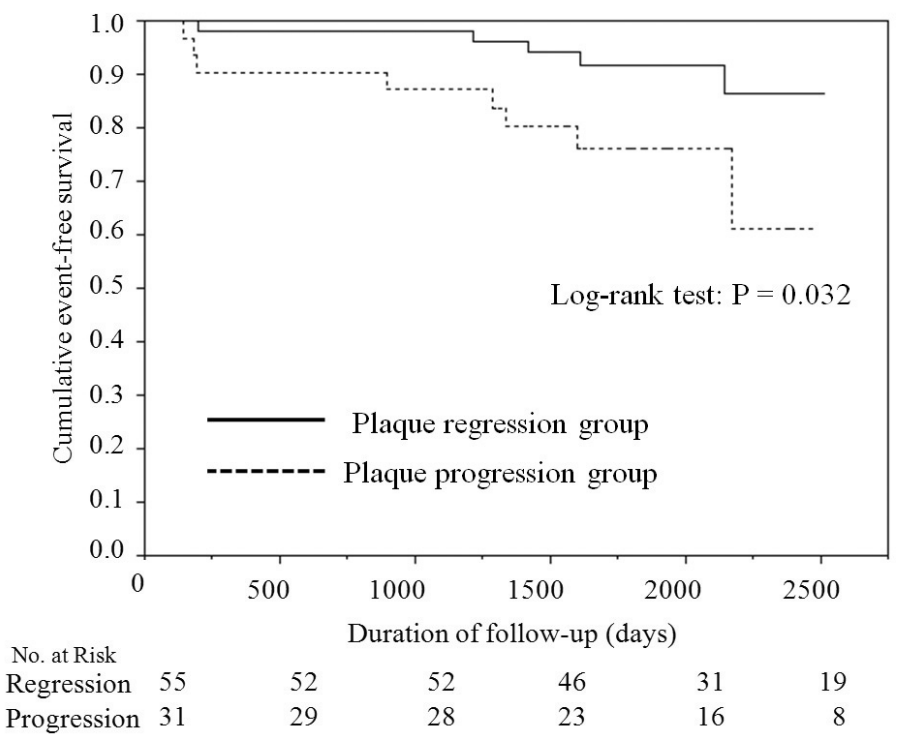

Fig. 3. Kaplan-Meier estimates of incidence of MACCE.

Cumulative event-free survival was significantly higher in regression group than in progression group (Log-rank test, $p=0.032$ ).

regression was significantly associated with MACCE (HR, 0.31; 95\% CI, 0.09 to $0.94 ; p=0.038$ ). The Cox proportional hazards model adjusted for plaque regression, age, gender, BMI, hypertension, diabetes and type of ACS revealed that plaque regression was significantly predictive for MACCE (HR, 0.26; $95 \%$ CI, 0.07 to $0.83 ; p=0.023$; Table 4). In addition, when a change in plaque volume was entered in the multivariate Cox proportional hazards model as a con- tinuous variable, a reduction in plaque volume tended to be good predictor of MACCE $(10 \%$ reduction in coronary plaque volume: HR 0.69, 95\%CI 0.46-1.01, $p=0.057)$.

\section{Discussion}

The present study showed that short-term plaque regression determined by IVUS is associated with a 
Table 4. Results of Cox proportional hazard regression analysis

\begin{tabular}{|c|c|c|c|c|c|c|}
\hline \multicolumn{7}{|c|}{ Proportional hazard model } \\
\hline \multirow{2}{*}{ Variables } & \multicolumn{3}{|c|}{ Unadjusted model } & \multicolumn{3}{|c|}{ Adjusted model ${ }^{*}$} \\
\hline & HR & $95 \% \mathrm{CI}$ & $p$ value & HR & $95 \% \mathrm{CI}$ & $p$ value \\
\hline Plaque regression - yes & 0.31 & $0.09-0.94$ & 0.038 & 0.26 & $0.07-0.83$ & 0.023 \\
\hline Age (1-year increase) & 1.03 & $0.97-1.09$ & 0.394 & 1.03 & $0.96-1.11$ & 0.412 \\
\hline Female-yes & 1.10 & $0.24-3.61$ & 0.887 & 1.11 & $0.22-4.61$ & 0.884 \\
\hline BMI (increase of 1) & 1.12 & $0.94-1.33$ & 0.225 & 1.11 & $0.91-1.36$ & 0.304 \\
\hline Hypertension-present & 1.64 & $0.53-6.07$ & 0.394 & 1.85 & $0.49-8.12$ & 0.371 \\
\hline Diabetes-present & 1.22 & $0.39-3.69$ & 0.713 & 0.88 & $0.25-2.88$ & 0.826 \\
\hline Type of ACS-AMI & 1.20 & $0.39-4.43$ & 0.756 & 1.26 & $0.39-4.86$ & 0.710 \\
\hline
\end{tabular}

BMI, body mass index; ACS, acute coronary syndrome; AMI, acute myocardial infarction. $p<0.05$ was considered significant.

* Multivariate Cox model included plaque regression, age, gender, BMI, hypertension, diabetes and type of ACS as variables.

lower rate of future cardiovascular events in patients with ACS. Furthermore, this association remained significant even after adjustment for clinically important covariates. To our knowledge, this is the first study to evaluate the impact of plaque regression on the longterm prognosis of patients with ACS.

Our IVUS findings showed that both plaque and vessel volumes significantly decreased in the regression group, whereas the quantity of vessels increased with increasing plaque volume in the progression group. Atherosclerosis primarily affects the arterial wall, with atherosclerotic plaque initially growing within an outwardly expanding vessel wall (positive remodeling) ${ }^{14)}$. Our findings indicated that the remodeling processes in progressive and regressive patients were positive and negative, respectively. Owing to this process, disease progression is not angiographically detectable during the early stages of plaque accumulation when the increasing total number of vessels occupies an increasing proportion of the plaque mass. Although angiographically determined disease progression reflects clinical prognosis, the present study found that changes in quantitative reference angiographic findings were similar between the two groups, whereas IVUS identified plaque regression or progression ${ }^{15,16)}$. Therefore, evaluating coronary vessel remodeling by angiography can be difficult after ACS, whereas IVUS, which can reveal arteriosclerotic changes, is more versatile. Changes in plaque measured by IVUS should lead to early risk stratification of patients after ACS over the short term.

We also believe that the targets of plaque regression are similar to those of vulnerable plaque stabilization. Vulnerable plaque results in recurrent coronary vascular events more often among patients with ACS than with stable angina ${ }^{17)}$. We speculate that intensive lipid management of vulnerable plaque composed of various lipid constituents would result in regression compared with stable plaque. Plaques that are prone to rupture are also most likely to regress ${ }^{18)}$. A CT angiographic study showed that patients with positively remodeled coronary lesions accompanied by low attenuation plaque are at higher risk for $\mathrm{ACS}^{19)}$. In addition, 39.5\% of patients with ACS had other coronary plaque lesions derived from a culprit lesion related to the event ${ }^{20)}$. Vulnerable plaque in non-target vessels is reportedly an important predictor of future critical cardiac events in IVUS studies ${ }^{21)}$; therefore, plaque regression in another coronary lesion could lead to the suppression of other events. We also considered that clinical outcome would be a more desirable and valid endpoint than simple plaque burden. Nicholls et al. recently reported results from six large serial IVUS imaging trials involving 4137 patients with established stable coronary artery disease who were followed up for an average of 21 months $^{22}$. They identified a direct relationship between the burden of coronary atherosclerosis, its progression, and adverse cardiovascular events. We considered that the results of that pooled analysis revealed the prognostic significance of changes in stable plaque, whereas our findings reflect the significance of changes in unstable plaque. We thus consider that the findings of both studies demonstrate the importance of plaque progression or regression in serial IVUS studies to the clinical outcomes of patients with coronary artery disease. Consequently, the notion that coronary plaque regression could lead to an improved prognosis supports previous results.

Surrogate IVUS endpoints have recently been applied in several trials of treatment regimens based on statins for patients with coronary artery disease ${ }^{4,5)}$. 
These studies identified a close linear relationship between the degree of LDL-C reduction and changes in plaque volume. Furthermore, pleiotropic statins evidently improve endothelial dysfunction, inhibit inflammatory responses, stabilize atherosclerotic plaques and diminish smooth muscle cell accumulation and collagen deposition ${ }^{18,23)}$. These effects could explain the regression of atherosclerosis. Plaque regressed in about $80 \%$ of the patients enrolled in the present study who had been treated with atorvastatin. As a matter of course, we consider that the administration of statins to lower LDL-C levels is the most effective strategy for treating atherosclerotic changes such as plaque regression. We similarly postulate that blood pressure plays an important role in the promotion of coronary artery disease. Chhatriwalla et al. found the most attenuation of coronary plaque progression in patients with very low LDL-C ( $\leq 70$ $\mathrm{mg} / \mathrm{dL}$ ) together with normal systolic blood pressure $(\leq 120 \mathrm{mmHg})^{24)}$. The results of another large pooled analysis also found that despite achieving intensive control of LDL-C, plaque continued to progress in $20 \%$ of patients with coronary artery disease ${ }^{25}$. That report also showed that these patients were more likely to progress if they had diabetes, greater increases in systolic blood pressure and smaller increases in HDL-C. Thus, we believe that these results not only provide important information about the effects of LDL-C and blood pressure on cardiovascular disease but also support the significance of multifactorial treatment for global risk in patients with coronary artery diseases including ACS.

Several limitations are associated with this study. Firstly, only 86 patients with ACS participated. Although our data are still useful because we evaluated prognosis from a novel viewpoint, further large-scale studies are warranted. Secondly, we used grayscale IVUS, so plaque composition was not evaluated; however, we believe that a reduction in the quantity of plaque approximates plaque stabilization. Future studies should evaluate the value of information generated from novel imaging techniques such as integrated backscatter and virtual histology, and whether these methods can detect changes in plaque composition as well as in plaque volume. Thirdly, we could not precisely evaluate the association among control of blood pressure, plaque volume change and clinical prognosis in the present study. Hence, the effects of multifactorial treatment, including cholesterol and blood pressure management, require further investigation to assess the prognostic significance of plaque regression.

In conclusion, plaque regression determined with volumetric IVUS over a period of 6 months predicted good long-term outcomes for patients with ACS. Furthermore, plaque regression could be a surrogate marker of prognosis from the early stage of ACS, while treatment aimed at plaque regression might improve long-term prognosis.

\section{Acknowledgements}

The authors are grateful to the staff of the Department of Cardiovascular Medicine at Juntendo University. We also thank Natsuko Yamamoto for IVUS data analysis, as well as Yumi Nozawa for secretarial assistance.

\section{References}

1) Nissen SE, Yock P: Intravascular ultrasound: novel pathophysiological insights and current clinical applications. Circulation, 2001; 103: 604-616

2) Mintz GS, Nissen SE, Anderson WD, Bailey SR, Erbel R, Fitzgerald PJ, Pinto FJ, Rosenfield K, Siegel RJ, Tuzcu EM, Yock PG: American College of Cardiology Clinical Expert Consensus Document on Standards for Acquisition, Measurement and Reporting of Intravascular Ultrasound Studies (IVUS). A report of the American College of Cardiology Task Force on Clinical Expert Consensus Documents. J Am Coll Cardiol, 2001; 37: 1478-1492

3) Nissen SE, Tsunoda T, Tuzcu EM, Schoenhagen P, Cooper CJ, Yasin M, Eaton GM, Lauer MA, Sheldon WS, Grines CL, Halpern S, Crowe T, Blankenship JC, Kerensky R. Effect of recombinant ApoA-I Milano on coronary atherosclerosis in patients with acute coronary syndromes: a randomized controlled trial. Jama, 2003; 290: 2292-2300

4) Nissen SE, Tuzcu EM, Schoenhagen P, Brown BG, Ganz P, Vogel RA, Crowe T, Howard G, Cooper CJ, Brodie B, Grines CL, DeMaria AN: Effect of intensive compared with moderate lipid-lowering therapy on progression of coronary atherosclerosis: a randomized controlled trial. Jama, 2004; 291: 1071-1080

5) Nissen SE, Nicholls SJ, Sipahi I, Libby P, Raichlen JS, Ballantyne CM, Davignon J, Erbel R, Fruchart JC, Tardif JC, Schoenhagen P, Crowe T, Cain V, Wolski K, Goormastic M, Tuzcu EM: Effect of very high-intensity statin therapy on regression of coronary atherosclerosis: the ASTEROID trial. Jama, 2006; 295: 1556-1565

6) Nissen SE, Nicholls SJ, Wolski K, Nesto R, Kupfer S, Perez A, Jure H, De Larochelliere R, Staniloae CS, Mavromatis K, Saw J, Hu B, Lincoff AM, Tuzcu EM: Comparison of pioglitazone vs glimepiride on progression of coronary atherosclerosis in patients with type 2 diabetes: the PERISCOPE randomized controlled trial. Jama, 2008; 299: 1561-1573

7) Hiro T, Kimura T, Morimoto T, Miyauchi K, Nakagawa $Y$, Yamagishi M, Ozaki Y, Kimura K, Saito S, Yamaguchi T, Daida H, Matsuzaki M: Effect of intensive statin therapy on regression of coronary atherosclerosis in patients with acute coronary syndrome: a multicenter randomized trial evaluated by volumetric intravascular ultrasound using 
pitavastatin versus atorvastatin (JAPAN-ACS [Japan assessment of pitavastatin and atorvastatin in acute coronary syndrome] study). J Am Coll Cardiol, 2009; 54: 293-302

8) Arai H, Hiro T, Kimura T, Morimoto T, Miyauchi K, Nakagawa Y, Yamagishi M, Ozaki Y, Kimura K, Saito S, Yamaguchi T, Daida H, Matsuzaki M: More Intensive Lipid Lowering is Associated with Regression of Coronary Atherosclerosis in Diabetic Patients with Acute Coronary Syndrome. J Atheroscler Thromb, 2010

9) Rodriguez-Granillo GA, Agostoni P, Garcia-Garcia HM, Biondi-Zoccai GG, McFadden E, Amoroso G, de Jaegere P, Bruining N, de Feyter P, Serruys PW: Meta-analysis of the studies assessing temporal changes in coronary plaque volume using intravascular ultrasound. Am J Cardiol, 2007; 99: 5-10

10) Okazaki S, Yokoyama T, Miyauchi K, Shimada K, Kurata T, Sato H, Daida H: Early statin treatment in patients with acute coronary syndrome: demonstration of the beneficial effect on atherosclerotic lesions by serial volumetric intravascular ultrasound analysis during half a year after coronary event: the ESTABLISH Study. Circulation, 2004; 110: 1061-1068

11) Dohi T, Miyauchi K, Okazaki S, Yokoyama T, Yanagisawa N, Tamura H, Kojima T, Yokoyama K, Kurata T, Daida H: Early intensive statin treatment for six months improves long-term clinical outcomes in patients with acute coronary syndrome (Extended-ESTABLISH trial): a follow-up study. Atherosclerosis, 2010; 210: 497-502

12) Karha J, Murphy SA, Kirtane AJ, de Lemos JA, Aroesty JM, Cannon CP, Antman EM, Braunwald E, Gibson $\mathrm{CM}$ : Evaluation of the association of proximal coronary culprit artery lesion location with clinical outcomes in acute myocardial infarction. Am J Cardiol, 2003; 92: 913-918

13) Executive Summary of The Third Report of The National Cholesterol Education Program (NCEP) Expert Panel on Detection, Evaluation, And Treatment of High Blood Cholesterol In Adults (Adult Treatment Panel III). Jama, 2001; 285: 2486-2497

14) Glagov S, Weisenberg E, Zarins CK, Stankunavicius R, Kolettis GJ: Compensatory enlargement of human atherosclerotic coronary arteries. N Engl J Med, 1987; 316: 1371-1375

15) Mack WJ, Xiang M, Selzer RH, Hodis HN: Serial quantitative coronary angiography and coronary events. Am Heart J, 2000; 139: 993-999
16) Azen SP, Mack WJ, Cashin-Hemphill L, LaBree L, Shircore AM, Selzer RH, Blankenhorn DH, Hodis HN: Progression of coronary artery disease predicts clinical coronary events. Long-term follow-up from the Cholesterol Lowering Atherosclerosis Study. Circulation, 1996; 93: 34-41

17) Fuster V, Badimon L, Badimon JJ, Chesebro JH: The pathogenesis of coronary artery disease and the acute coronary syndromes (1). N Engl J Med, 1992; 326: 242-250

18) Fukumoto Y, Libby P, Rabkin E, Hill CC, Enomoto M, Hirouchi Y, Shiomi M, Aikawa M: Statins alter smooth muscle cell accumulation and collagen content in established atheroma of watanabe heritable hyperlipidemic rabbits. Circulation, 2001; 103: 993-999

19) Motoyama S, Sarai M, Harigaya $H$, Anno H, Inoue $K$, Hara T, Naruse H, Ishii J, Hishida H, Wong ND, Virmani R, Kondo T, Ozaki Y, Narula J: Computed tomographic angiography characteristics of atherosclerotic plaques subsequently resulting in acute coronary syndrome. J Am Coll Cardiol, 2009; 54: 49-57

20) Goldstein JA, Demetriou D, Grines CL, Pica M, Shoukfeh M, O'Neill WW: Multiple complex coronary plaques in patients with acute myocardial infarction. $\mathrm{N}$ Engl J Med, 2000; 343: 915-922

21) Okura H, Kobayashi Y, Sumitsuji S, Terashima M, Kataoka T, Masutani M, Ohyanagi M, Shimada K, Taguchi H, Yasuga Y, Takeda Y, Ohashi Y, Awano K, Fujii K, Mintz GS: Effect of culprit-lesion remodeling versus plaque rupture on three-year outcome in patients with acute coronary syndrome. Am J Cardiol, 2009; 103: 791-795

22) Nicholls SJ, Hsu A, Wolski K, Hu B, Bayturan O, Lavoie A, Uno K, Tuzcu EM, Nissen SE: Intravascular ultrasound-derived measures of coronary atherosclerotic plaque burden and clinical outcome. J Am Coll Cardiol, 2010; 55: 2399-2407

23) Davignon J: Beneficial cardiovascular pleiotropic effects of statins. Circulation, 2004; 109: III 39-43

24) Chhatriwalla AK, Nicholls SJ, Wang TH, Wolski K, Sipahi I, Crowe T, Schoenhagen P, Kapadia S, Tuzcu EM, Nissen SE: Low levels of low-density lipoprotein cholesterol and blood pressure and progression of coronary atherosclerosis. J Am Coll Cardiol, 2009; 53: 1110-1115

25) Bayturan O, Kapadia S, Nicholls SJ, Tuzcu EM, Shao M, Uno K, Shreevatsa A, Lavoie AJ, Wolski K, Schoenhagen P, Nissen SE: Clinical predictors of plaque progression despite very low levels of low-density lipoprotein cholesterol. J Am Coll Cardiol, 2010; 55: 2736-2742 